\title{
Spin state in the propagation of quantum relativistic particles along classical trajectories
}

\author{
Jesús Rubio and Alfredo Luis* \\ Departamento de Óptica, Facultad de Ciencias Físicas, Universidad Complutense, 28040 Madrid, Spain
}

(Received 2 February 2014; revised manuscript received 27 April 2014; published 22 May 2014)

\begin{abstract}
We address the propagation of the spin along classical trajectories for a spin-1/2 particle obeying the Dirac equation with scalar potentials. Focusing on classical trajectories as the exact propagation of wave-function discontinuities, we find an explicit spin-transport law for the case of the Dirac oscillator. In the general case we examine the spin propagation along classical trajectories emerging as an approximation of the quantum dynamics via the mechanical analog of the optical eikonal asymptotic approach. Throughout we establish as many parallels as possible with the equivalent situation for the electromagnetic field.
\end{abstract}

DOI: 10.1103/PhysRevA.89.052128

PACS number(s): 03.65.Ca, 03.65.Sq

\section{INTRODUCTION}

In the theory of light the deep relationship between electromagnetic and geometrical optics is well known. Usually, geometrical optics is considered as an approximation valid in the limit of short wavelengths [1,2]. Furthermore, another radically different point of view is also possible: the light rays of geometrical optics determine the exact way in which the surfaces of field discontinuity propagate, for all wavelengths $[3,4]$. In this context, a natural question appears: What happens with the polarization? A simple calculus shows a coupling between the rays of light, the gradient of the refraction index, and the field itself; consequently, it can be proved that the ellipse of polarization just rotates along the propagation, always keeping its shape [3,4]. It is a remarkable phenomenon that the polarization transformation has a purely geometrical origin since it depends only on the shape of the trajectory, becoming an example of topological dynamics.

In this work we explore the behavior of the spin state of a spin- $1 / 2$ particle when its spinorial wave function is transported along classical trajectories, in the same sense that we understand the transport of polarization for a photon. As in the optical case, we can follow two road maps.

On the one hand, we may regard the classical trajectories as the exact way in which any surface of discontinuity of the spinorial wave function must propagate [5], as recalled in Sec. II A. For spin-1/2 particles in purely scalar potentials, the conclusion of Ref. [5] was that the propagation of discontinuities provides no useful information about the spin transport along the classical trajectories. Thus we introduce an explicitly relativistic implementation of a classical scalar potential involving spin-dependent terms. This is the case of the Dirac oscillator [6]. The corresponding propagation of spin discontinuities along the classical trajectories is carried out in Sec. II B.

On the other hand, we may consider that classical trajectories also emerge in the quantum-mechanical version of the optical eikonal approach. This actually corresponds to the Wentzel-Kramers-Brillouin (WKB) methods [7], where the classical trajectories arise as an approximation of quantum dynamics valid for small values of the Planck constant. The spin propagation along these classical trajectories is carried

\footnotetext{
*alluis@ fis.ucm.es; http://www.ucm.es/info/gioq
}

out in Sec. III. To this end we mimic the optical case as far as possible. In order to exploit those parallels we recall the eikonal approach for Maxwell's equations in the Appendix.

\section{QUANTUM-DISCONTINUITY APPROACH}

\section{A. Formalism}

For the sake of completeness, we recall here the basic tools required to address the propagation of discontinuities in optics as well as in quantum mechanics, as presented in Ref. [5]. For both situations we will consider that the evolution is given by the solution of a system of linear partial differential equations,

$$
\sum_{\mu, v} \frac{\partial}{\partial x^{\mu}}\left(M_{j}^{\mu, v} \psi_{\nu}\right)=0
$$

where $x^{\mu}=x, y, z, t$ are the space-time coordinates, $M_{j}^{\mu, \nu}$ are functions of $x^{\eta}$, and $\psi_{v}\left(x^{\mu}\right)$ are the Cartesian components either of a spinorial quantum wave function or of a classical electromagnetic field.

As discussed in Ref. [3], Eqs. (2.1) are conditions for the components $\psi_{v}$ at every point where they are continuous, but they cannot establish conditions for the boundary values of $\psi_{v}$ on a surface of discontinuity. Therefore, in order to deal with discontinuities it is advantageous to replace Eqs. (2.1) by their integral counterparts. To this end we consider volume integrals of Eq. (2.1), which can then be suitably converted into surface integrals using the divergence theorem [3],

$$
\int_{\Gamma} d \Gamma \sum_{\mu, v} \frac{\partial}{\partial x^{\mu}}\left(M_{j}^{\mu, v} \psi_{\nu}\right)=\sum_{\mu, v} \int_{\gamma} d \gamma_{\mu} M_{j}^{\mu, v} \psi_{\nu}=0
$$

where $d \Gamma=d x d y d z d t$ is the differential of four-dimensional volume and $d \gamma_{\mu}$ are the Cartesian components of the surface element normal to the three-dimensional surface $\gamma$ enclosing $\Gamma$. The last equality in Eq. (2.2) is fully equivalent to Eq. (2.1) when $\psi_{v}$ are continuous. On the other hand Eq. (2.2) is more general since it can be applied without difficulties when $\psi_{v}$ are discontinuous.

Our aim is to derive conditions for the discontinuities of $\psi_{v}$ by imposing Eq. (2.2). Denoting by $S(\boldsymbol{x}, t)=0$ the surface of discontinuity, we apply Eq. (2.2) to two volumes $\Gamma_{1}$ and $\Gamma_{2}$ connected by $S$, as well as to the whole volume $\Gamma_{1}+\Gamma_{2}$ (see 


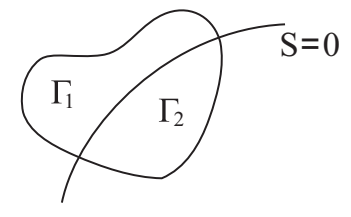

FIG. 1. Diagram illustrating the volumes $\Gamma_{1}$ and $\Gamma_{2}$ joined by the surface of discontinuity $S=0$.

Fig. 1). This leads to

$$
\sum_{\mu, v}\left[\psi_{v}\right] \frac{\partial S}{\partial x^{\mu}} M_{j}^{\mu, v}=0,
$$

where for each $\boldsymbol{x}, t$ we have that $\left[\psi_{v}\right]$ denotes the difference between the boundary values of $\psi_{\nu}$ at the two sides of $S$, i.e., $\left[\psi_{\nu}\right]=\psi_{\nu}\left(\right.$ at $\left.\Gamma_{2}\right)-\psi_{\nu}\left(\right.$ at $\left.\Gamma_{1}\right)$. We have considered $d \gamma_{\mu} \propto$ $\partial S / \partial x^{\mu}$ and that $\Gamma_{1,2}$ are arbitrary. Moreover, we assume that $M_{j}^{\mu, \nu}$ are continuous at $S$. Equations (2.3) are the conditions we were looking for.

In particular, when this formalism is applied to the Dirac equation with scalar potential $V(\boldsymbol{x})$ and definite fixed energy $E$, i.e., for harmonic wave functions, we get [5]

$$
E=\sqrt{c^{2}(\nabla W)^{2}+m^{2} c^{4}}+V,
$$

having introduced a separation of variables such that $S(x, t)=$ $W(\boldsymbol{x})-E t$. This means that the surface $S$ where the discontinuity of harmonic wave functions takes place must satisfy the classical Hamilton-Jacobi equation for the relativistic Hamiltonian $H=\sqrt{c^{2} \boldsymbol{p}^{2}+m^{2} c^{4}}+V$. Therefore, the evolution of $S$ is fully determined by the classical trajectories for the problem [8], so we say that $S$ follows the classical trajectories. The result is exact since no approximation nor limiting procedure whatsoever has been used.

\section{B. Dirac oscillator}

It is worth noting that for scalar potentials the formalism outlined above provides no information about the spin transport along the classical trajectories. As mentioned in the Introduction, we will address this issue within the quantumdiscontinuity approach to classical trajectories considering the relativistic formulation of scalar potentials including spindependent terms. This is the case of the Dirac oscillator [6].

The Dirac oscillator is a simple model for a relativistic isotropic oscillator of frequency $\omega$ and mass $m$, which preserves the fully linear character of the Dirac equation,

$$
i \hbar \frac{\partial \boldsymbol{\Psi}}{\partial t}=c(\boldsymbol{p} \cdot \boldsymbol{\alpha}-i m \omega \boldsymbol{x} \cdot \boldsymbol{\alpha} \beta+m c \beta) \boldsymbol{\Psi},
$$

where $\boldsymbol{p}=-i \hbar \nabla, \boldsymbol{\alpha}$ and $\beta$ are the $4 \times 4$ matrices

$$
\boldsymbol{\alpha}=\left(\begin{array}{ll}
0 & \sigma \\
\sigma & 0
\end{array}\right), \quad \beta=\left(\begin{array}{cc}
I & 0 \\
0 & -I
\end{array}\right),
$$

$\sigma$ are the three Pauli matrices, $I$ is the $2 \times 2$ identity, and the spinorial four-dimensional wave function $\boldsymbol{\Psi}$ depends on the Cartesian coordinates $\boldsymbol{x}$ and time $t$.

At this stage we cannot apply the previous formalism directly because the lack of partial derivatives in the terms $(-i m \omega \boldsymbol{x} \cdot \boldsymbol{\alpha} \beta+m c \beta) \boldsymbol{\Psi}$ impedes the expression of Eq. (2.5) in the form (2.1). As in classical optics we can avoid this difficulty by restricting our analysis to time-harmonic wave functions for which

$$
\boldsymbol{\Psi}=\frac{i \hbar}{E} \frac{\partial \boldsymbol{\Psi}}{\partial t}
$$

so that Eq. (2.5) can be rewritten as

$$
\frac{\partial \boldsymbol{\Psi}}{\partial t}=-c \boldsymbol{\alpha} \cdot \nabla \boldsymbol{\Psi}+\frac{c}{E} \frac{\partial}{\partial t}[(-i m \omega \boldsymbol{x} \cdot \boldsymbol{\alpha} \beta+m c \beta) \boldsymbol{\Psi}]
$$

Since all terms now have partial derivatives we can apply Eq. (2.3), leading to

$[\boldsymbol{\Psi}] \frac{\partial S}{\partial t}=-c \boldsymbol{\nabla} S \cdot \boldsymbol{\alpha}[\boldsymbol{\Psi}]+\frac{c}{E} \frac{\partial S}{\partial t}(-i m \omega \boldsymbol{x} \cdot \boldsymbol{\alpha} \beta+m c \beta)[\boldsymbol{\Psi}]$,

where $[\Psi]$ represents again the difference between the boundary values of $\boldsymbol{\Psi}$ at the two sides of a certain surface $S(\boldsymbol{x}, t)$ where this discontinuity takes place.

For the sake of clarity, this four-dimensional equation can be split into a pair of two-dimensional equations using Eq. (2.6),

$$
\begin{aligned}
\left(m c^{2}-E\right) \frac{\partial S}{\partial t}[\boldsymbol{\phi}] & =c\left(E \nabla S-i \frac{\partial S}{\partial t} m \omega \boldsymbol{x}\right) \cdot \boldsymbol{\sigma}[\boldsymbol{\chi}], \\
-\left(m c^{2}+E\right) \frac{\partial S}{\partial t}[\chi] & =c\left(E \nabla S+i \frac{\partial S}{\partial t} m \omega \boldsymbol{x}\right) \cdot \boldsymbol{\sigma}[\boldsymbol{\phi}],
\end{aligned}
$$

where the two-dimensional spinors $\phi$ and $\chi$ are defined as

$$
\boldsymbol{\Psi}=\left(\begin{array}{l}
\phi \\
\chi
\end{array}\right) \text {. }
$$

Using one of Eqs. (2.10) to remove the discontinuity of the lower spinor $[\chi]$, and taking into account the general relation

$$
(\boldsymbol{A} \cdot \boldsymbol{\sigma})(\boldsymbol{B} \cdot \boldsymbol{\sigma})=(\boldsymbol{A} \cdot \boldsymbol{B}) I+i(\boldsymbol{A} \times \boldsymbol{B}) \boldsymbol{\sigma},
$$

we get a two-dimensional equation for the discontinuity of the upper spinor $[\boldsymbol{\phi}]$

$$
\begin{aligned}
\left(\frac{\partial S}{\partial t}\right)^{2}\left(E^{2}-m^{2} c^{4}\right)[\boldsymbol{\phi}]= & c^{2}\left[E^{2}(\nabla S)^{2}+\left(\frac{\partial S}{\partial t}\right)^{2} m^{2} \omega^{2} \boldsymbol{x}^{2}\right. \\
& \left.+2 m E \omega \frac{\partial S}{\partial t}(\boldsymbol{x} \times \nabla S) \cdot \boldsymbol{\sigma}\right][\boldsymbol{\phi}] .
\end{aligned}
$$

This equation is of the form

$$
(x \times \nabla S) \cdot \sigma[\phi]=\lambda[\phi],
$$

so that $\lambda= \pm|\boldsymbol{x} \times \nabla S|$. Hence we must have

$$
\begin{aligned}
& \left(\frac{\partial S}{\partial t}\right)^{2} \frac{E^{2}-m^{2} c^{4}}{E^{2} c^{2}}-(\nabla S)^{2}-\frac{1}{E^{2}}\left(\frac{\partial S}{\partial t}\right)^{2} m^{2} \omega^{2} x^{2} \\
& \pm \frac{2 m \omega}{E} \frac{\partial S}{\partial t}|\boldsymbol{x} \times \nabla S|=0 .
\end{aligned}
$$

This is the equation that $S(\boldsymbol{x}, t)$ must satisfy in order to describe a surface of discontinuity for the quantum wave function of a Dirac oscillator. Note that actually there is no sign freedom in Eq. (2.15) because there is no sign ambiguity in Eq. (2.13). 


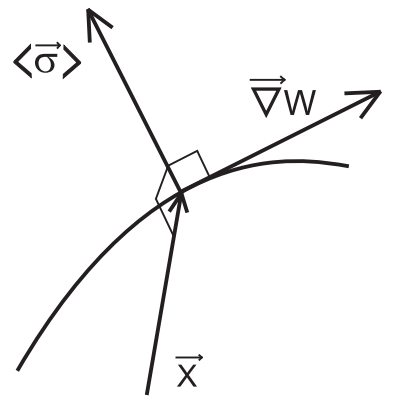

FIG. 2. Diagram illustrating the relation between the tangent $\nabla W$ to the trajectory $\boldsymbol{x}$, and the spin state represented by the vector $\langle\boldsymbol{\sigma}\rangle$, which is always orthogonal to both $\nabla W$ and $\boldsymbol{x}$.

Since the Hamiltonian we are considering is time independent and the wave function is harmonic, it is natural to use the method of separation of variables to separate out the time in the form $S(\boldsymbol{x}, t)=W(\boldsymbol{x})-E t$, leading to a much simpler equation for $W(\boldsymbol{x})$,

$$
\frac{E^{2}-m^{2} c^{4}}{c^{2}}-(\nabla W)^{2}-m^{2} \omega^{2} \boldsymbol{x}^{2} \mp 2 m \omega|\boldsymbol{x} \times \nabla W|=0 .
$$

Equations (2.15) and (2.16) can be readily interpreted as the Hamilton-Jacobi equations for the Dirac oscillator, and their solutions are the corresponding classical trajectories where $\nabla W$ is normal to the discontinuity surface and tangent to the trajectory at each point. These trajectories are the same plane ellipses of the standard oscillator since the extra term $x \times \nabla W$ just depends on the orbital angular momentum that is a constant of the motion.

The key point for our purposes here is that the eigenvalue equation (2.13) determines a single definite spin state at each point so it explicitly contains how the spin is transported along the classical trajectories. More specifically, the spin state is always an eigenstate of the spin projection along the direction of the orbital angular momentum $\boldsymbol{x} \times \nabla W$, i.e., normal to the plane where the trajectory is contained (see Fig. 2). Thus we may say that the spin does not influence the trajectory while the trajectory forces the spin state. Therefore, it seems that the topological properties of the photon-polarization transport are lost in the spin-1/2 case.

Finally we stress that this explicit spin evolution for the discontinuity holds because of the presence of a term proportional to $(\boldsymbol{x} \times \nabla W) \cdot \boldsymbol{\sigma}$ in (2.13), which is a strong spinorbit coupling that is also preserved in the the nonrelativistic limit [6]. Nevertheless, the physical system being described is a harmonic oscillator whose potential is scalar.

\section{EIKONAL APPROACH}

The Dirac oscillator allowed us to determine the evolution of the spin state by applying the formalism of the propagation of discontinuities with no approximation. However, for physical situations with purely scalar potentials the divergence theorem does not provide enough information to determine the spin evolution along trajectories. To avoid this obstacle we consider the emergence of classical trajectories as the quantum-mechanical version of the optical eikonal. This is the WKB method [7]. In this work we are particularly interested in applying this method to determine the transport of the spin along such classical trajectories. We put particular emphasis on preserving as far as possible the parallelism with the electromagnetic case, in particular regarding its simplicity.

\section{A. Transport equation}

Let us start with the Dirac equation for a spin-1/2 particle within an arbitrary scalar potential $V(\boldsymbol{x})$, with definite energy $E$, mass $m$, and a time-harmonic wave function

$$
\left[-i \hbar c \boldsymbol{\alpha} \cdot \nabla+m c^{2} \beta+V(\boldsymbol{x})\right] \boldsymbol{\Psi}=E \boldsymbol{\Psi} .
$$

Instead of considering spin-dependent potentials, now we address a more transparent approach just in terms of $V(\boldsymbol{x})$, mimicking as far as possible the electromagnetic situation where light evolution is governed by the scalar position-dependent quantities $\epsilon(\boldsymbol{x})$ and $\mu(\boldsymbol{x})$. Splitting the four-dimensional spinor $\boldsymbol{\Psi}$ as in Eq. (2.11), and using Eq. (2.6), the four-dimensional matrix equation (3.1) can be decoupled into a pair of two-dimensional equations, as follows:

$$
\begin{aligned}
& -i \hbar c(\boldsymbol{\sigma} \cdot \nabla) \chi=\left(E-m c^{2}-V\right) \boldsymbol{\phi}, \\
& -i \hbar c(\boldsymbol{\sigma} \cdot \nabla) \boldsymbol{\phi}=\left(E+m c^{2}-V\right) \chi .
\end{aligned}
$$

These two equations share roughly the structure of the second pair of Maxwell's equations in Eq. (A2), via the rough correspondences

$$
\hbar \leftrightarrow 1 / k_{0}, \quad \boldsymbol{\phi}, \boldsymbol{\chi} \leftrightarrow \boldsymbol{E}, \boldsymbol{H}, \quad V(\boldsymbol{x}) \leftrightarrow \mu(\boldsymbol{x}), \epsilon(\boldsymbol{x}) .
$$

However, in the Dirac case there is no counterpart of the first two Maxwell equations (A1). This is to say that matter waves lack the transversality condition satisfied by light waves, so we may expect some basic differences regarding the vectorial behavior.

Continuing with the analogy, we construct now a kind of second-order differential wave equation for the Dirac case equivalent to Eq. (A3). This is always possible by clearing $\chi$ from the second equation of Eq. (3.2) and substituting it into the first one:

$$
\begin{aligned}
\hbar^{2} \nabla^{2} \boldsymbol{\phi} & +\left[\left(\frac{E-V}{c}\right)^{2}-m^{2} c^{4}\right] \boldsymbol{\phi}+\frac{\hbar^{2} \nabla V \cdot \nabla}{E-V+m c^{2}} \boldsymbol{\phi} \\
& +i \frac{\hbar^{2} \boldsymbol{\sigma} \cdot[\nabla V \times \nabla]}{E-V+m c^{2}} \boldsymbol{\phi}=0 .
\end{aligned}
$$

Developing this further, we apply the eikonal approximation $\hbar \rightarrow 0$ as an analog of the electromagnetic Eq. (A4), looking for solutions of the form

$$
\boldsymbol{\phi}=\left(\boldsymbol{\phi}_{0}+\hbar \boldsymbol{\phi}_{1}+\cdots\right) e^{i \tilde{S} / \hbar},
$$

where $\tilde{S}(\boldsymbol{x}, t)$ is the phase of the wave function. We are using the same symbol used above for a surface of discontinuity for the reasons shown below. Substituting Eq. (3.5) into Eq. (3.4) and considering again the separation of variables $\tilde{S}(\boldsymbol{x}, t)=$ $\tilde{W}(\boldsymbol{x})-E t$ for a time-independent Hamiltonian and harmonic wave functions, we get the following result for the order $\hbar^{0}$, 
after some lengthy but straightforward algebra:

$$
[\nabla \tilde{W}(\boldsymbol{x})]^{2}=\left[\frac{E-V(\boldsymbol{x})}{c}\right]^{2}-m^{2} c^{4} .
$$

This is the analog of an eikonal equation independent of spin for the electron, fully analogous to Eq. (A5) of the electromagnetic case. Comparing Eqs. (3.6) and (A5), it is very remarkable that the only real difference between them is the fact that the former has mass.

Note that this eikonal approach leads us to an equation for the phase $\tilde{S}$ that is formally identical to the classical HamiltonJacobi equation for the relativistic Hamiltonian (2.4). So we may say that $\tilde{S}$ represents the classical action of the system. Moreover, note that the phase $\tilde{S}$ here and the surface of discontinuity $S$ in Sec. II obey exactly the same dynamics as expressed by Eqs. (2.4) and (3.6), so deep down they represent the same physical entity but emerging under slightly different approaches. This is the reason why we use closely related symbols for both quantities. Moreover, from now on we take $\tilde{W}=W$ for the sake of simplicity.

Roughly speaking, the equivalence between $\tilde{S}$ and $S$ holds since when $\hbar \rightarrow 0$ the phase $\tilde{S} / \hbar$ of the quantum wave function in Eq. (3.5) becomes effectively discontinuous at every point of the space by displaying very large variations in very short displacements. On the other hand, we now introduce the expansion (3.5) in Eq. (3.4), and again after some lengthy but straightforward algebra, the order $\hbar^{1}$ of Eq. (3.4) is

$$
\frac{d}{d s} \boldsymbol{\phi}_{0}=-\frac{\nabla^{2} W}{2|\nabla W|} \phi_{0}-\frac{(\nabla V \cdot \sigma)(\nabla W \cdot \boldsymbol{\sigma})}{2\left(E+m c^{2}-V\right)|\nabla W|} \boldsymbol{\phi}_{0},
$$

where we have used that the derivative along the arclength $s$ of the trajectory reads

$$
\frac{d}{d s}=\frac{\nabla W \cdot \nabla}{|\nabla W|} .
$$

After relation (2.12), this is equivalent to

$$
\begin{aligned}
\frac{d}{d s} \boldsymbol{\phi}_{0}= & -\frac{\nabla^{2} W}{2|\nabla W|} \boldsymbol{\phi}_{0}-\frac{\nabla V \cdot \nabla W}{2\left(E+m c^{2}-V\right)|\nabla W|} \boldsymbol{\phi}_{0} \\
& -i \frac{(\nabla V \times \nabla W) \cdot \boldsymbol{\sigma}}{2\left(E+m c^{2}-V\right)|\nabla W|} \boldsymbol{\phi}_{0} .
\end{aligned}
$$

This is precisely the transport equation we were looking for. As we announced, it shares exactly the same structure as the electromagnetic counterpart in Eq. (A6) since both are linear equations of the form $d \boldsymbol{A} / d s=M \boldsymbol{A}$. The method of coupling of the physical objects in each case is, however, different. In the Maxwell transport, the electromagnetic field is a pair of three-dimensional vectors coupled with the gradient of the inhomogeneous parameters $\nabla \epsilon$ and $\nabla \mu$, as well as with the tangent to the trajectory $\nabla L$. These are all three-dimensional vectors. Meanwhile, Dirac transport vectorially couples the tangent to the trajectory $\nabla W$, the gradient of the scalar potential $\nabla V$, and the spin $\sigma$. However, now the fermionic field, which is divided into two bispinors, has a spinorial four-dimensional character, so it cannot be specifically coupled on the same grounds with the other dynamical objects $\nabla V$ and $\nabla W$, which are three-dimensional vectors.

Note that in the nonrelativistic limit $E-m c^{2} \ll m c^{2}$ the spin-trajectory coupling between $\sigma$ and $\nabla W$ disappears. Thus spin transport in the absence of magnetic fields is a purely relativistic phenomenon.

\section{B. Amplitude and spin}

The general transport equation (3.9) can be split into equations for amplitude $\left|\phi_{0}(\boldsymbol{x})\right|$ and local spin state $\boldsymbol{u}_{0}(\boldsymbol{x})$ after decomposing $\boldsymbol{\phi}$ in the form

$$
\boldsymbol{\phi}_{0}=\left|\boldsymbol{\phi}_{0}\right| \boldsymbol{u}_{0}, \quad\left|\boldsymbol{u}_{0}\right|=1 .
$$

For the amplitude $\left|\phi_{0}\right|$ we get

$$
\frac{d}{d s}\left|\boldsymbol{\phi}_{0}\right|=-\left(\frac{\nabla^{2} W}{2|\nabla W|}+\frac{\nabla V \cdot \nabla W}{2\left(E+m c^{2}-V\right)|\nabla W|}\right)\left|\boldsymbol{\phi}_{0}\right|,
$$

while for the spin state $\boldsymbol{u}_{0}$ we have

$$
\frac{d}{d s} \boldsymbol{u}_{0}=-i \frac{(\nabla V \times \nabla W) \cdot \boldsymbol{\sigma}}{2\left(E+m c^{2}-V\right)|\nabla W|} \boldsymbol{u}_{0} .
$$

In particular this last expression allows us to derive a transport equation for the local mean value $\langle A\rangle$ of any spin observable $A$ as

$$
\langle A\rangle=\boldsymbol{u}_{0}^{\dagger} A \boldsymbol{u}_{0}, \quad \frac{d}{d s}\langle A\rangle \propto-i\langle[(\nabla V \times \nabla W) \cdot \boldsymbol{\sigma}, A]\rangle,
$$

and in particular

$$
\frac{d}{d s}\langle\boldsymbol{\sigma}\rangle \propto\langle\boldsymbol{\sigma}\rangle \times(\nabla V \times \nabla W) .
$$

By local spin mean values we mean that $\langle A\rangle$ depends on $\boldsymbol{x}$. This shows that the spin transport is made of consecutive local rotations around the vector $\nabla V \times \nabla W$. In particular, the projection of the spin on the vector $\nabla V \times \nabla W$ is constant $\langle(\nabla V \times \nabla W) \cdot \sigma\rangle=$ const (see Fig. 3).

We can particularize to the harmonic oscillator $V(\boldsymbol{x}) \propto \boldsymbol{x}^{2}$ in order to compare with the result for the Dirac oscillator above. In such a case the trajectories are in the plane defined by $\nabla V$ and $\nabla W$. Nevertheless, unlike the Dirac-oscillator case the spin state need not be constant and orthogonal to the trajectory plane, and its evolution will depend in general on the

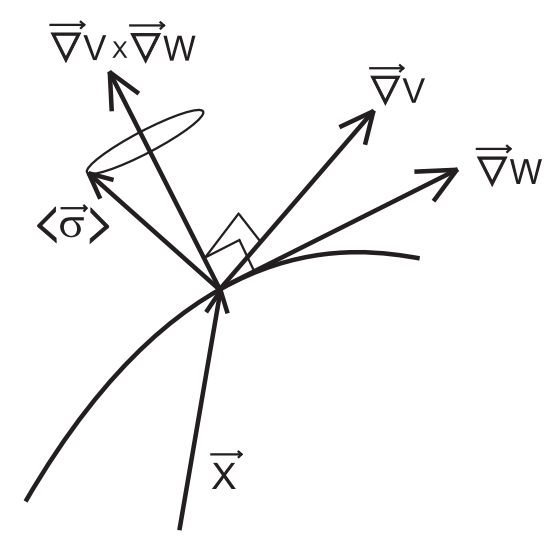

FIG. 3. Diagram illustrating the evolution of the spin state represented by the vector $\langle\boldsymbol{\sigma}\rangle$ rotating around the axis $\nabla V \times \nabla W$ where $\nabla W$ is tangent to the trajectory $\boldsymbol{x}$ and $\nabla V$ is the gradient of the potential. 
trajectory followed. Moreover, the transport equation depends on dynamical features other than the form of the trajectory, so seemingly also in this case the geometrical character of the transport is lost in the transition from light to matter.

\section{APPLICATIONS}

We explore now the application of the above formalism. The most trivial example is the case of a free particle. In such a case $\nabla V=\mathbf{0}$ so that the spin is constant along the trajectory. Thus, to obtain simple illustrative and nontrivial results we focus on two further examples: planar circular and circular helicoidal trajectories. In particular, the second one admits a direct comparison with similar applications of the optical eikonal regarding the emergence of geometric phases [4].

\section{A. Planar circular trajectory}

The simplest nontrivial example is provided by a planar circular trajectory for a particle moving in a three-dimensional central potential $V=V\left(\sqrt{x^{2}+y^{2}+z^{2}}\right)$, with

$$
x=r_{0} \cos \left(s / r_{0}\right), \quad y=r_{0} \sin \left(s / r_{0}\right), \quad z=0,
$$

where $s$ is the arclength parameter, $r_{0}$ being the radius of the trajectory. The corresponding tangent vector is

$$
\frac{\nabla W}{|\nabla W|}=(-y, x, 0) / r_{0},
$$

while $\nabla V=-k(x, y, 0)$, for a suitable constant $k$ depending in general on $r_{0}$. Therefore, the spin-transport equation (3.12) becomes

$$
\frac{d}{d s} \boldsymbol{u}_{0}=i \frac{k r_{0}}{2\left(E+m c^{2}-V_{0}\right)} \sigma_{z} \boldsymbol{u}_{0},
$$

where $V_{0}=V\left(r_{0}\right)$. The solution is rather simple,

$$
\boldsymbol{u}_{0}(s)=e^{i \theta \sigma_{z}} \boldsymbol{u}_{0}(0), \quad \theta=\frac{k r_{0} s}{2\left(E+m c^{2}-V_{0}\right)} .
$$

The result is a spin rotation around the axis $z$ of angle $\theta$ (see Fig. 4).

It is worth noting that when the particle completes a revolution of arclength $s_{0}=2 \pi r_{0}$ the spin does not return to its initial state $\boldsymbol{u}_{0}\left(s_{0}\right) \neq \boldsymbol{u}_{0}(0)$. This would be a kind of geometrical phase if it were not for the fact that the phase acquired in closed loops does not depend only on the form of the trajectory, but also on dynamical factors represented

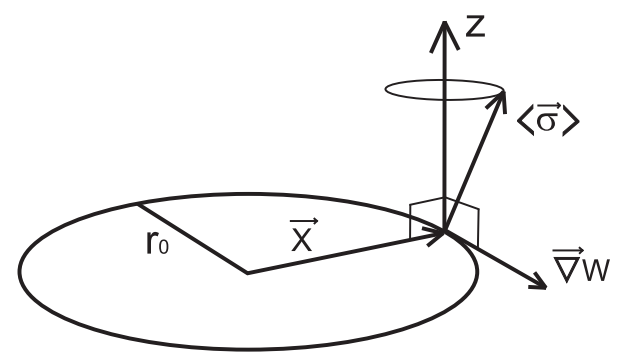

FIG. 4. Diagram illustrating the evolution of the spin state represented by the vector $\langle\sigma\rangle$ rotating around the axis $z$ normal to the plane of the trajectory. by $k$ and $V_{0}$. Next we discuss this point with a more detailed example.

\section{B. Circular helicoidal trajectory}

Let us consider a circular helicoidal trajectory

$$
x=r_{0} \cos (\Omega z), \quad y=r_{0} \sin (\Omega z)
$$

where the arclength is $s=\sqrt{1+\Omega^{2} r_{0}^{2}}, r_{0}$ is the radius, and the helix pitch is $2 \pi / \Omega$. The tangent to the trajectory reads

$$
\frac{\nabla W}{|\nabla W|}=\frac{(-\Omega y, \Omega x, 1)}{\sqrt{1+\Omega^{2} r_{0}^{2}}} .
$$

We assume that this motion in the plane $x, y$ takes place in a two-dimensional central potential $V=V\left(\sqrt{x^{2}+y^{2}}\right)$, so that $\nabla V=-k(x, y, 0)$, for a suitable constant $k$ that depends on $r_{0}$. Otherwise the particle is free to move along the $z$ axis, which is the helix axis. Taking into account that

$$
\frac{\nabla V \times \nabla W}{|\nabla W|}=\frac{k\left(-y, x, \Omega r_{0}^{2}\right)}{\sqrt{1+\Omega^{2} r_{0}^{2}}}
$$

the spin-transport equation (3.12) becomes

$$
\frac{d}{d s} \boldsymbol{u}_{0}=i \mu\left(y \sigma_{x}-x \sigma_{y}+\Omega r_{0}^{2} \sigma_{z}\right) \boldsymbol{u}_{0},
$$

where

$$
\mu=\frac{k / \sqrt{1+\Omega^{2} r_{0}^{2}}}{2\left(E+m c^{2}-V_{0}\right)}, \quad V_{0}=V\left(r_{0}\right) .
$$

This can be easily solved using standard techniques for this kind of problem, leading to

$$
\boldsymbol{u}_{0}(s)=e^{-i \delta \sigma_{z}} e^{i\left[(\delta+\theta) \sigma_{z}-\varphi \sigma_{y}\right]} \boldsymbol{u}_{0}(0),
$$

with

$$
\delta=\frac{\Omega s}{2 \sqrt{1+\Omega^{2} r_{0}^{2}}}, \quad \theta=\mu r_{0}^{2} \Omega s, \quad \varphi=\mu r_{0} s / 2 .
$$

For all practical purposes the rest-mass energy is the largest factor so that $E+m c^{2}-V_{0} \simeq 2 m c^{2}$. Therefore

$$
\mu \simeq \frac{k / \sqrt{1+\Omega^{2} r_{0}^{2}}}{2 m c^{2}}
$$

and we can regard $\mu$ as well as $\theta$ and $\varphi$ as very small parameters. This allows a power series expansion of the exact result (4.10) in powers of $\theta$ and $\varphi$, retaining just the first order,

$$
\boldsymbol{u}_{0}(s) \simeq\left[\sigma_{0}+i \theta \sigma_{z}+\varphi \frac{\sin \delta}{\delta}\left(\sigma_{+} e^{-i \delta}-\sigma_{-} e^{i \delta}\right)\right] \boldsymbol{u}_{0}(0)
$$

where $\sigma_{0}$ is the identity matrix, and $\sigma_{ \pm}=\sigma_{x} \pm i \sigma_{y}$.

We can appreciate that after a helix pitch of arclength $s_{0}$ with $\Omega s_{0}=2 \pi \sqrt{1+\Omega^{2} r_{0}^{2}}$, the spin $\boldsymbol{u}_{0}\left(s_{0}\right)$ does not return to its original state $\boldsymbol{u}_{0}(0)$, but

$$
\boldsymbol{u}_{0}(s)=\left(\sigma_{0}+i \theta \Omega \sigma_{z}\right) \boldsymbol{u}_{0}(0) \simeq e^{i \theta \sigma_{z}} \boldsymbol{u}_{0}(0),
$$




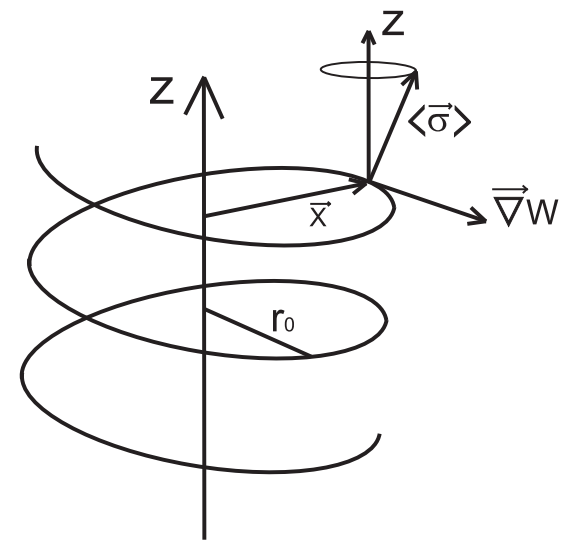

FIG. 5. Diagram illustrating the evolution of the spin state represented by the vector $\langle\boldsymbol{\sigma}\rangle$ rotating around the $z$ axis, which is the axis of the helix.

having taken into account that for $s=s_{0}$ we have $\delta=\pi$. This is a simple small rotation around the $z$ axis (see Fig. 5), with angle

$$
\theta \simeq \frac{\pi k r_{0}^{2}}{m c^{2}}
$$

This result strongly recalls the geometric phase arising in the optical case of polarization transport along a helix [4], which also results in a rotation of polarization around the $z$ axis of the Poincaré sphere (circular polarization being at the poles). However, there is a strong difference since in our case the spin transformation after a helix pitch does not depend just on the form of the trajectory, given by $\Omega$ and $r_{0}$. In addition, it depends also on dynamical features represented by the factor $k$, depending on the particular potential leading to such trajectory.

We can try to develop further the optical-mechanical analogy by considering that the central potential is a harmonic oscillator $V=k\left(x^{2}+y^{2}\right) / 2$. Denoting by $v_{z}$ the speed along the helix axis we have $z=v_{z} t$ and $\Omega z=\Omega v_{z} t=\sqrt{k / m} t$ so that $k=m \Omega^{2} v_{z}^{2}$. After Eqs. (4.14) and (4.15) the angle rotated by the spin after a helix pitch is

$$
\theta \simeq \pi \Omega^{2} r_{0}^{2} \frac{v_{z}^{2}}{c^{2}}
$$

This can be compared with the angle through which the light polarization is rotated per pitch for the same helix, which is $2 \pi / \sqrt{1+\Omega^{2} r_{0}^{2}}$. We appreciate that the dependence on $\Omega r_{0}$ is different, and that in the case of matter and in the mechanical case there is a factor $v_{z} / c$, which is typically small.

\section{CONCLUSIONS}

We have studied the behavior of the spin state of a spin-1/2 particle when its spinorial wave function is transported along classical trajectories. As happens in classical electromagnetism, classical trajectories can emerge within a wavelike picture via two procedures: from the exact propagation of discontinuities and from the eikonal approximation.

It is known that the propagation of discontinuities for the Dirac equation with purely scalar potentials (i.e., the analogous situation for light in isotropic and inhomogeneous media) does not provide enough information about the spin of the particle. Then, a first option is to introduce an explicitly relativistic implementation of a classical scalar potential involving spindependent terms: the Dirac oscillator. This approach has shown a definite relation between the spin discontinuity and the tangent to the classical trajectory, which is an exact solution.

For an arbitrary scalar potential, we cannot obtain exact results for the spin propagation. Thus we turn our attention to the approximate picture provided by WKB methods, establishing as many clear parallels as possible with the equivalent and known situation for the electromagnetic field. In this way we have been able to find the equation of the spin transport for Dirac particles. This result reveals three main differences regarding the Maxwell asymptotic approach: (i) The electromagnetic field satisfies orthogonality relations (A1) that are absent in the Dirac case, (ii) there is a mass term difference in the Dirac eikonal (3.6) in comparison with the Maxwell case (A5), and (iii) in the Dirac case there is no projection of spin along the tangent to the trajectory in Eq. (3.12), a difference with the Maxwell case (A12). These differences might explain the lack of spin-propagation effects in the nonrelativistic limit, and the lack of topological features in the matter case. In particular, we have applied this theory to two nontrivial cases, circular and helicoidal trajectories, providing two simple examples for the general results of the main discussion.

\section{ACKNOWLEDGMENTS}

A.L. acknowledges support from Projects No. FIS201235583 of the Spanish Ministerio de Economía y Competitividad and No. QUITEMAD S2009-ESP-1594 of the Consejería de Educación de la Comunidad de Madrid.

\section{APPENDIX: EIKONAL APPROACH FOR THE ELECTROMAGNETIC FIELD}

We review here the eikonal approach for the electromagnetic field to be compared with the Dirac case. The Maxwell field equations for time-harmonic waves of frequency $\omega$ within isotropic and inhomogeneous media are

$$
\begin{gathered}
\nabla[\epsilon(\boldsymbol{x}) \boldsymbol{E}(\boldsymbol{x}, t)]=0, \quad \nabla[\mu(\boldsymbol{x}) \boldsymbol{H}(\boldsymbol{x}, t)]=0, \\
-\frac{i}{k_{0}} \nabla \times \boldsymbol{E}(\boldsymbol{x}, t)=\mu(\boldsymbol{x}) c \boldsymbol{H}(\boldsymbol{x}, t), \\
\frac{i}{k_{0}} \nabla \times \boldsymbol{H}(\boldsymbol{x}, t)=\epsilon(\boldsymbol{x}) c \boldsymbol{E}(\boldsymbol{x}, t),
\end{gathered}
$$

where $k_{0}=\omega / c$. They can be combined to get the vectorial wave equation

$$
\nabla^{2} \boldsymbol{E}+n^{2} k_{0}^{2} \boldsymbol{E}+\nabla\left(\frac{1}{\epsilon} \nabla \epsilon \cdot \boldsymbol{E}\right)+\frac{1}{\mu} \nabla \mu \times(\nabla \times \boldsymbol{E})=0,
$$

where $n(\boldsymbol{x})=c \sqrt{\epsilon(\boldsymbol{x}) \mu(\boldsymbol{x})}$ is the refraction index.

To apply the eikonal approximation in the limit $1 / k_{0} \rightarrow 0$ we consider asymptotic solutions of the form [2]

$$
\boldsymbol{E}=\left(\boldsymbol{E}_{0}+\frac{1}{k_{0}} \boldsymbol{E}_{1}+\cdots\right) e^{i k_{0} S^{\prime}},
$$


where $S^{\prime}(\boldsymbol{x}, t)=L(\boldsymbol{x})-c t$ is the phase. Introducing Eq. (A4) into Eq. (A3) we get for the lowest $1 / k_{0}^{0}$ order

$$
[\nabla L(\boldsymbol{x})]^{2}=n^{2}(\boldsymbol{x}),
$$

which is the well-known eikonal equation.

For the $1 / k_{0}^{1}$ order we have

$\frac{d}{d s} \boldsymbol{E}_{0}=-\frac{\nabla^{2} L}{2|\nabla L|} \boldsymbol{E}_{0}-\frac{\left(\boldsymbol{E}_{0} \cdot \nabla \epsilon\right) \nabla L}{2 \epsilon|\nabla L|}-\frac{\nabla \mu \times\left(\nabla L \times \boldsymbol{E}_{0}\right)}{2 \mu|\nabla L|}$,

where

$$
\frac{d}{d s}=\frac{\nabla L \cdot \nabla}{|\nabla L|}
$$

is the derivation with respect to the arclength $s$. The last term in Eq. (A6) can be expressed also as

$$
\frac{\nabla \mu \times\left(\nabla L \times \boldsymbol{E}_{0}\right)}{2 \mu|\nabla L|}=\frac{\left(\boldsymbol{E}_{0} \cdot \nabla \mu\right) \nabla L}{2 \mu|\nabla L|}-\frac{(\nabla L \cdot \nabla \mu) \boldsymbol{E}_{0}}{2 \mu|\nabla L|} .
$$

This is the transport equation that expresses the propagation of the amplitude and polarization of the photon along the trajectories determined by the eikonal equation (A5).

On the other hand, if we insert Eq. (A4) directly in Eq. (A1) we get again Eq. (A5) plus the explicit relations of orthogonality

$$
\boldsymbol{E}_{0} \cdot \nabla L=0, \quad \boldsymbol{H}_{0} \cdot \nabla L=0 .
$$

The transport equation (A6) can be further split into propagation equations for the local amplitude $\left|\boldsymbol{E}_{0}\right|$ and the local polarization state $\boldsymbol{u}_{0}$, both depending on $\boldsymbol{x}$, after decomposing $\boldsymbol{E}_{0}$ in the form

$$
\boldsymbol{E}_{0}=\left|\boldsymbol{E}_{0}\right| \boldsymbol{u}_{0}, \quad\left|\boldsymbol{u}_{0}\right|=1 .
$$

This leads to the following equation of propagation for the local amplitude $\left|\boldsymbol{E}_{0}\right|$ :

$$
\frac{d}{d s}\left|\boldsymbol{E}_{0}\right|=-\left(\frac{\nabla^{2} L}{2|\nabla L|}-\frac{\nabla L \cdot \nabla \mu}{2 \mu|\nabla L|}\right)\left|\boldsymbol{E}_{0}\right|,
$$

and to this one for the local polarization state $\boldsymbol{u}_{0}$ :

$$
\frac{d}{d s} \boldsymbol{u}_{0}=-\frac{\left(\boldsymbol{u}_{0} \cdot \nabla \epsilon\right) \nabla L}{2 \epsilon|\nabla L|}-\frac{\left(\boldsymbol{u}_{0} \cdot \nabla \mu\right) \nabla L}{2 \mu|\nabla L|} .
$$

[1] M. Born and E. Wolf, Principles of Optics (Cambridge University Press, Cambridge, 1998)

[2] Y. A. Kravtsov and Y. I. Orlov, Geometrical Optics of Inhomogeneous Media (Springer-Verlag, Heidelberg, 1990).

[3] R. K. Luneburg, Mathematical Theory of Optics (University of California Press, Berkeley, 1966).

[4] E. Bortolotti, Atti R. Accad. Naz. Lincei Rend. Cl. Sci. Fis. Mat. Nat. 4, 552 (1926); J. N. Ross, Opt. Quantum Electron. 16, 455 (1984); F. D. M. Haldane, Opt. Lett. 11, 730 (1986); A. Tomita and R. Y. Chiao, Phys. Rev. Lett. 57, 937 (1986); J. Liñares, M. C. Nistal, and D. Baldomir, Appl. Opt. 33, 4293 (1994).

[5] A. Luis, Phys. Rev. A 67, 024102 (2003).
[6] M. Moshinsky and A. Szczepaniak, J. Phys. A 22, L817 (1989); P. Strange, Relativistic Quantum Mechanics (Cambridge University Press, Cambridge, 1998); E. Sadurni, in Symmetries in Physics, edited by R. Bijker, O. Castaños, R. Jáuregui, and O. Rosas-Ortiz, AIP Conference Proceedings No. 1334 (AIP, New York, 2011), pp. 249-290.

[7] S. I. Rubinow and J. B. Keller, Phys. Rev. 131, 2789 (1963); J. Bolte and S. Keppeler, Phys. Rev. Lett. 81, 1987 (1998); Ann. Phys. (N.Y.) 274, 125 (1999); H. Spohn, ibid. 282, 420 (2000).

[8] E. J. Saletan and A. H. Cromer, Theoretical Mechanics (John Wiley and Sons, New York, 1971). 